\title{
Oscillatory eddy structure in a container
}

\section{By P. N. SHANKAR, R. KIDAMBIAND J. HARIHARA N}

\author{
Computational \& Theoretical Fluid Dynamics Division. \\ National Aerospace Laboratories, Bangalore 5600 17. India \\ pns@ctfd.cmmacs.ernet.in
}

(Received 10 February 2003 and in revised form 16 June 2003)

We consider the periodic, two-dimensional motion of a viscous, incompressible liquid which fills a rectangular container. The motion is due to the periodic motion of the lid which moves in its own plane. If the velocities are sufficiently small the motion will be governed by the linearized Navier-Stokes equations and consequently the dimensionless stream function $\Psi(x, z, t)=\psi(x, z) \mathrm{e}^{\mathrm{e} t}$ will satisfy the equation $\nabla^{4} \psi-$ $\mathrm{iRe} \nabla^{2} \psi=0$, where $\operatorname{Re}$ is the Reynolds number. If we then seek separable solutions for $\psi(x, z)$ that satisfy the no-slip conditions on the sidewalls, it is easy to show that the problem reduces to the eigenvalue problem

$$
A \tan \frac{1}{2} \lambda=\sqrt{\lambda^{2}-i R e} \tan \frac{1}{2} \sqrt{\lambda^{2}-i R e}
$$

where $\lambda$ is the eigenvalue. A detailed analysis is made of this eigenvalue problem. All the eigenvalues are complex; all eigenvalues with positive real part either belong to a set $\left\{\lambda_{n}^{u}\right\}$ in the upper half-plane or to another $\left\{\lambda_{n}^{I}\right\}$ in the lower half-plane. They satisfy the important relationship $\lambda_{n}^{l}=\sqrt{\lambda_{n}^{u}+i R e}$. We show by an asymptotic analysis that while the $\lambda_{n l}^{l}$ move to the neighbourhood of the real axis as $R e \rightarrow \infty$, the A" move away from the origin and approach the line $\lambda_{i}=\lambda_{r}$ in the complex- $\lambda_{\text {-plane. This fact has }}$ an important bearing on the damping of gravity waves at high Reynolds numbers. The eigenfunctions derived above are used to write down a formal expansion for the stream function and the coefficients are determined from the boundary conditions using a least-squares procedure. An examination of the resulting streamline patterns reveals interesting inertial effects even at low Reynolds numbers. In particular we examine the mechanism by which the flow field reverses its direction when the lid stops and reverses its direction of motion. If inertial effects are completely negelected, as has been done till now, one would infer an immediate response of the fluid to the changes in the lid motion; for example, one would conclude, wrongly, that when the lid is at rest so is the fluid. Our analysis shows, in fact, a very intricate and beautiful mechanism, involving among other things an apparent engulfing of the corner eddy by the new primary eddy, by which the direction of the circulation is reversed in the fluid. These results should be of importance in the analysis of mixing, where such effects appear to have been ignored till now.

\section{Introduction}

There is a large body of work dealing with the technologically important problem of mixing, where the fluid motion is driven by the periodic motion of one or more of the boundaries of the container. In most of these studies, see for example Ottino (1989, 1990), the fluid velocity field at low Reynolds numbers is found from experiments 
but the mixing characteristics are determined by analysing the time evolution of a blob released at some time and place in the fluid. These analyses are usually based on ideas and techniques from nonlinear dynamical systems. It is well-known that mixing is greatly enhanced by periodic rather than steady motions of the walls; there are some beautiful and instructive pictures in Ottino (1989) that illustrate this. On the other hand, there is an even larger body of work on the steady velocity fields generated in closed containers by the steady motion of one or more of the bounding walls (Shankar \& Deshpande 2000). This body of work is mostly computational since a large range of Reynolds numbers is covered but it also contains some important experimental and analytical or semi-analytical work at the lower Reynolds numbers. There appears to be very little work, if any, dealing with periodic motions in lid-driven cavities or containers. Very recently a number of papers have appeared (Galaktionov et al. 1999; Anderson et al. 1999; Malyuga et al. 2002) that consider, using analytical and computational tools, the mixing problem in containers with time-periodic stirring protocols. However, in these papers all the inertial terms are dropped when dealing with Stokes flow and effectively the fields are quasi-steady rather than truly unsteady.

As a consequence potentially important unsteady effects from the $d v / d t$ term in the momentum equation have been lost.

The problem that we consider here is a generalized version of the Stokes first problem (Batchelor 1967) which considers flow in a semi-infinite half-space due to an oscillating plane boundary. The only non-zero velocity component in the Stokes problem is the one parallel to the plate; the velocity profile can be described as a damped transverse wave propagating in a direction perpendicular to the plane boundary. The velocity variation damps out rapidly before it can diffuse in a given time with the result that the motion is essentially confined to a 'penetration depth'. The presence of side- and bottom walls changes the picture considerably. In the presence of the bottom wall alone, the velocity profile can be described as being due to a system of transverse waves rather than a single one. The single most important contribution of the sidewalls is the system of eddies - in the corners as well as in the bulk of the fluid.

The only paper that we are aware of that deals with the unsteady Stokes or similar equations in a partially confined geometry is Sturges \& Joseph (1977). They consider the motion in a simple fluid, i.e. a non-Newtonian fluid, between two infinite planes generated by the tangential, in-plane oscillatory motion of one of the planes. The fluid has a free surface between the planes and is unbounded below. Sturges \& Joseph develop a perturbation expansion for the field in terms of $\epsilon$, the amplitude of the oscillatory plate motion, and the field is determined to leading order giving an estimate for the perturbed free-surface height, the quantity of primary interest to the authors. It is interesting to note that no rise occurs if the motion of the sidewall is steady. Also of interest is that the analysis leads to an equation for the stream function that is identical in form to the one that follows from the linearized Navier-Stokes equations. Sturges \& Joseph solve this equation using an eigenfunction expansion procedure.

In this paper we consider the simplest case of the periodic motion of a Newtonian fluid completely filling a container; the motion is generated by the simple harmonic oscillation of the lid. The container is assumed to be rectangular and the flow twodimensional. One could well ask whether any new and interesting features are likely to be found in this flow field. An interesting question of course is: how does the direction of the general circulation change when the lid reverses its direction of motion over a period? In the quasi-steady picture this change would take place immediately with no phase lag and no intermediate structures formed. But this is obviously incorrect and 
only an unsteady analysis can yield the correct physical picture. Another question pertains to the nature of the primary and corner eddies in unsteady flow and their dependence on the the container depth and the Reynolds number, a new parameter that now enters the problem. On the analytical side, there are interesting questions that arise regarding the distribution of the spatial eigenvalues in the complex plane: how does the spectrum differ from that obtaining in the steady case and how is its distribution affected by increasing Reynolds number? We will show in what follows that there are very interesting answers to all these questions, some of them quite unexpected.

We should also point out an important connection that this work has to the calculation of viscous damping of surface waves in a container. If capillary hysteresis at the contact line and viscous dissipation at the free surface due to surface contamination can be ignored, wave damping is only due to viscous dissipation in the wall boundary layers and in the bulk of the fluid. Traditionally, in order to avoid the complexity of a full viscous calculation, the latter have been estimated separately (see, for example, Case \& Parkinson 1957 and Miles 1967) and then added to estimate the total damping rates. More recently Nicolas (2002) has made a direct calculation of the total dissipation by solving for the full viscous field in the container with the appropriate boundary conditions at the liquid free surface. His method, involving the use of essentially real harmonic eigenfunctions, yields results that are in very good agreement with experimentally measured damping rates. In our opinion, it should be possible to more efficiently solve the full problem using the natural complex eigenfunctions of the linearized Navier-Stokes equations. The present work is a first step in this direction as it provides key information about the relevant spectrum, especially for large Reynolds numbers.

\section{Analysis}

\subsection{Formulation and non-dimensionalization}

Consider a rectangular container of width $L$ and depth $H$ completely filled with a Newtonian fluid of density $p$ and kinematic viscosity $v$. The lid of the container, assumed to make perfect contact with the fluid, oscillates in its own plane generating a velocity field in the fluid. We use a two-dimensional Cartesian coordinate system with $x$ aligned with the direction of the lid motion and with $z$ perpendicular to it and upwards. In the case of containers of finite depth the origin will be at the middle of the container; for a container of infinite depth the origin will be at the top of the container on the plane of symmetry. Let $\Omega$ be the frequency of the lid motion and $U$ be its maximum linear speed. In flow situations involving a translational velocity scale $U$ and a frequency $£ 2$ as is the case here, it is possible and natural (Happel \& Brenner 1973 ) to define a 'translational' Reynolds number $R e_{t r}=U L / v$ and an 'oscillatory' Reynolds number $R e_{t s}=\Omega L^{2} / \nu$. $R e_{t r}$ and $R e_{o s}$ can be varied independently by varying $U$ and $\Omega$. In the rest of the paper, we assume that the Strouhal number, $S r=R e_{o x} / R e_{t r}=\Omega L / U=L / a>1$; this permits us to drop the nonlinear convective terms in the momentum equations while retaining the unsteady terms. Note that here $a$ is the amplitude of the lid motion. We now non-dimensionalize all lengths by $L$, time by $\Omega^{-1}$. velocities by $U$ and the pressure by $\rho \nu U R e_{o s} / L$. Continuity and the linearized Navier-Stokes equations then take the form 


$$
v_{, t}=-\nabla p+\frac{1}{R e}{ }^{\jmath} v \text {. }
$$

Here $R e=L^{2} \Omega / \nu$ is the oscillatory Reynolds number of the flow. As in steady flows, it will be convenient to work with a scalar stream function $\Psi(x, z, t)$ such that $u=\psi$. and $w=-\Psi_{x}$ where $u$ and $w$ are the $x$-and $z$-componentsof the fluid velocityit follows then that the field is determined by the single scalar equation

$$
\nabla^{4} \Psi-\operatorname{Re} \nabla^{2} \Psi_{, t}=0
$$

Since the lid velocity has been assumed to be simple harmonic in time, we can assume that $\psi(x, z, t)=\psi(x, z) \mathrm{e}^{1 t}$ with the understanding that ultimately we will take the real part of the expression. Then the reduced stream function $\psi(x, z)$ satisfies the field equation

$$
\nabla^{4} \psi-\mathrm{i} R e \nabla^{2} \psi=0
$$

The no-slip boundary conditions require that $u=w=0$ on the sidewalls $x= \pm 1 / 2$ and the bottom wall $\mathrm{z}=-h / 2$; on the lid $z=h / 2, w=0$ and $u=u_{0}(x) \mathrm{e}^{\mathrm{it}}$. If the lid speed is uniform in $x, u_{0}(x)=1$; however it is advantageous to have a smooth distribution in $x$ vanishing at the end points as then the series that we use will converge very rapidly. Throughout this paper we choose for the lid speed

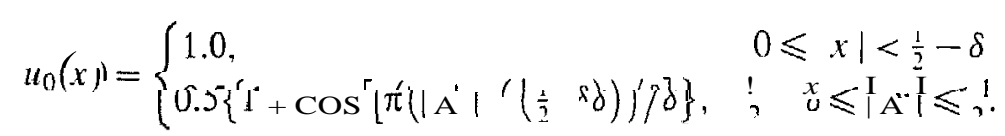

Thus $u_{0}(x)$ is just 1 over most of the lid but goes monotonically to zero near the sidewalls; throughout we take $S$ to be 0.1 . In summary, the boundary conditions on the reduced stream function are

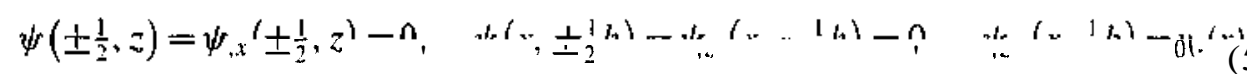

If we now seek solutions for $\psi$ of the form $f(x) \mathrm{e}^{\lambda z}, f(x)$ would have to satisfy

$$
f^{\mathrm{iv}}+\left(2 \lambda^{2}-\mathrm{i} R e\right) f^{\prime \prime}+\left(\lambda^{4}-\mathrm{i} \operatorname{Re} \lambda^{2}\right) f^{\prime}=0 .
$$

Since $\psi$ has to be symmetric in $x$ and the velocity has to vanish on the sidewalls we find that

$$
f(x) \sim \cos \lambda x+B \cos \sqrt{\lambda^{2}-i R} e x
$$

with the eigenvalues the roots of

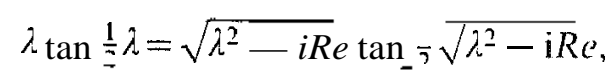

and the scalar $5=-\cos \frac{1}{2} \dot{\lambda} / \cos \frac{1}{2} \sqrt{\lambda^{2}} \overline{-i R e}$. The eigenvalue equation (8) arises in a different form and notation and "in a different context in Sturges \& Joseph (1977). We will examine this equation in some detail in the next sub-section.

\subsection{The distribution of the eigenvalues in the complex plane}

We observe that if we let $R e \rightarrow 0,(8)$ reduces to the well-studied and extensively used $\sin \lambda=-\lambda$, theequation governing steady cavityflows, anti-symmetric in $x$. The latter equation has only complex roots and an infinite number of them such that if $\mu$ is a root, then $-\mu$ and $\bar{\mu}$ are also roots; thus the roots are distributed symmetrically about both axes in the complex plane in this limiting case. That this will not be so in the present case when $R e \neq 0$ is indicated by the presence of the $\mathrm{i}$ in equation (8): 
(a)

$R e$

$\begin{array}{rc}0.1 & 4.21486+2.25535 \mathrm{i} \\ 1.0 & 4.23742+2.29724 \mathrm{j} \\ 10.0 & 4.49313+2.74167 \mathrm{i} \\ 100.0 & 7.65180+6.80733 \mathrm{i} \\ 500.0 & 16.01122+15.66115 \mathrm{i} \\ 1000.0 & 22.49233+22.25225 \mathrm{i} \\ 5000.0 & 50.05344+49.95081 \mathrm{i} \\ 10000.0 & 70.74760+70.67583 \mathrm{i} \\ & \end{array}$

(b) Re

$\begin{array}{rr}0.1 & 4.20993-2.24612 \mathrm{i} \\ 1.0 & 4.18809-2.20492 \mathrm{i} \\ 10.0 & 4.00197-1.82876 \mathrm{i} \\ 100.0 & 3.54365-0.58931 \mathrm{i} \\ 500.0 & 3.33744-0.22597 \mathrm{i} \\ 1000.0 & 3.28115-0.15384 \mathrm{i} \\ 5000.0 & 3.20435-0.06542 \mathrm{i} \\ 10000.0 & 3.18600-0.04571 \mathrm{i}\end{array}$

בz
$10.71316+3.10530 \mathrm{i}$
$10.71881+3.12476 \mathrm{i}$
$10.77893+3.32591 \mathrm{i}$
$11.73193+5.75383 \mathrm{i}$
$17.64269+14.56024 \mathrm{i}$
$23.56161+21.41564 \mathrm{i}$
$50.48265+49.55943 \mathrm{i}$
$71.04356+70.39783 \mathrm{i}$
$\lambda_{2}^{l}$
$10.71191-3.10100 \mathrm{i}$
$10.70634-3.08169 \mathrm{i}$
$10.65402-2.89560 \mathrm{i}$
$10.36266-1.68910 \mathrm{i}$
$9.98695-0.68908 \mathrm{i}$
$9.83552-0.46637 \mathrm{i}$
$9.61246-0.19673 \mathrm{i}$
$9.55779-0.13731 \mathrm{i}$

TABLE 1. (a) The first three eigenvalues A", A" and A" in the first quadrant as functions of the Reynolds number. (b) The first three eigenvalues $\bar{\lambda}_{1}^{l}, \lambda_{n}$ and $\lambda_{3}$ in the fourth quadrant as functions of the Reynolds number.

now if $\mathrm{A}$ is a root, $\overline{\mathrm{A}}$ is not necessarily a root. However, before discussing the detailed structure of the spectrum as a function of $R e$ we will, to fix ideas, first present some numerical results. Starting from the $R e-0$ limit the roots can be obtained using Newton's method; we use the principle of the argument to ensure that all roots in a given region of the complex plane have indeed been found. Figure 1 shows the first ten roots for three values of the Reynolds number. We will order the roots in the first and fourth quadrants in increasing values of their real parts. The roots in the first and fourth quadrants belong to the sets $\left\{\lambda_{n}^{u}, \mathrm{n}=1,2, \ldots\right\}$ and $\left\{\lambda_{n}^{l}, n=1,2, \ldots\right\}$ respectively. It can be observed from figure 1 that (i) $\lambda_{n}^{l} \neq \bar{\lambda}_{n}^{\prime \prime}$, (ii) as $n$ increases the spacing along the real axis tends to a constant value, $2 \pi$, and (iii) whereas the $\lambda_{n}^{u}$ move away from the real axis as $R e$ increases, the $\lambda_{n}^{l}$ move towards the real axis. Table 1 lists the first three eigenvalues, correct to all the places shown, over the range $0.1 \leqslant R e \leqslant 10,000$.

We now list some properties of the eigenvalues and derive their asymptotic forms.

(a) Equation (8) has solutions in all four quadrants of the complex plane. It is clear from $(8)$ that $-\lambda_{n}^{u}$ and $-\lambda_{n}^{\prime}$ are eigenvalues as well.

(b) For $R e=0$, (8) reduces to the corresponding relation $\sin \lambda=-\lambda$. This is shown by expanding the right-hand side of (8) in $R e$ and taking the limit $R e \rightarrow 0$.

(c) It is well known (Shankar 1993) that for $R e=0, \lambda_{n}^{\prime \prime}$ and $\lambda_{n}^{l}$ are complex conjugates. Although this does not hold here for $R e>0$ one might ask if an equivalent relationship holds. It is a straightforward matter to show that

$$
\lambda_{n}^{\prime}=\sqrt{\bar{\lambda}_{\|}^{\prime}+i R e}
$$

This also holds with the roles of $u$ and / interchanged. These relations are very important as now only one branch needs to be computed, with (9) giving the other.

(d) We now determine the asymptotic forms of the eigenvalues for $R e \rightarrow \infty$. Defining appropriate branch cuts through the branch points $\pm \mathrm{e}^{\mathrm{i} \pi / 4} \sqrt{R e}$ and 

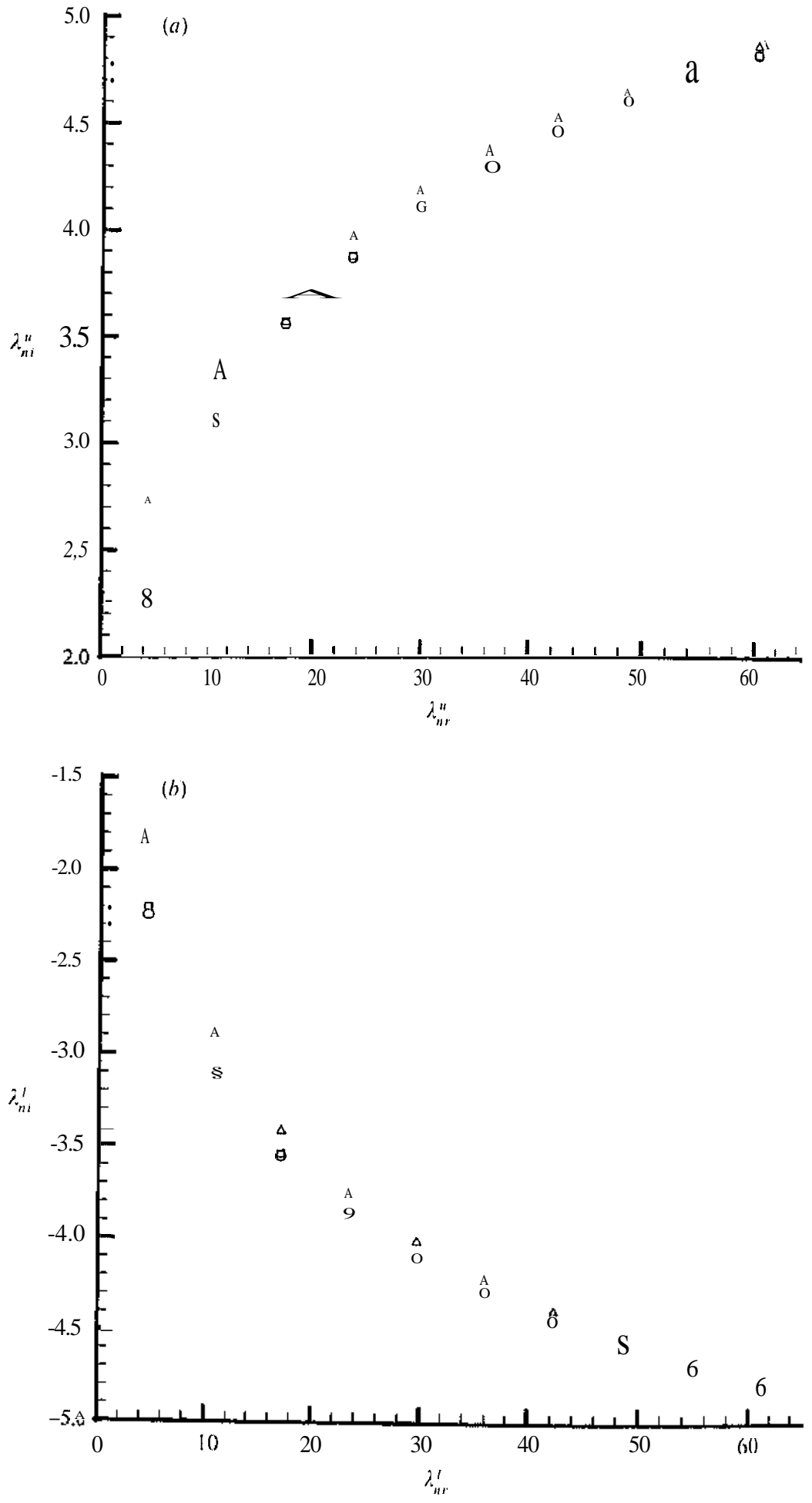

FIGURE 1. The distribution of the first ten eigenvalues. O $R \iota^{i}=0.1 ; \mathrm{n}, R \iota^{\prime}=1.0 ; \mathrm{A}, R e=10.0$. (a) $\lambda_{\mu \prime}^{\prime \prime},(b) \lambda_{\mu \prime}^{\prime}$. 
recognizing that $\lambda_{n}^{l}$ tend to the inviscid values in the infinite- $R \rho$ limit we assume $\lambda_{n}^{l}=(2 n-1) \pi+\lambda_{n 1}^{l}(R e)$. On substituting this form into (8) and equating the real and imaginary parts, we obtain

$$
\begin{aligned}
& \frac{2(2 n-1) \pi \lambda_{n ! r}^{l}}{\lambda_{n l i}^{l}+\lambda_{n 1 i}^{l}{ }^{2}}+2=\sqrt{\frac{R e}{2}} \\
& \frac{2(2 n-1) \pi \lambda_{n 1 i}^{l}}{i_{n 1 r}{ }^{3}+\lambda_{n 1 i}^{l}}=-\sqrt{\frac{R e}{2}}
\end{aligned}
$$

from which we obtain

$$
\begin{gathered}
\lambda_{n 1 r}^{\prime}-\frac{2(2 n-1) \pi \alpha(1-2 \alpha)}{1+(2 \alpha-1)^{2}} \\
\lambda_{n 1 i}^{\prime}=-\frac{2(2 n-1) \pi \alpha}{1+(2 \alpha-1)^{2}}
\end{gathered}
$$

with $\mathrm{a}=\sqrt{2} / \operatorname{Re} ; \lambda_{n 1,}^{l}$, and $\lambda_{n 1 i}^{l}$ are the real and imaginary parts of $\lambda_{n 1}^{l}$. Thus, in the limit $\operatorname{Re} \rightarrow \infty$

$$
\lambda_{n}^{\prime} \sim \frac{2(2 n-1) \pi}{11^{1}\left(2 \alpha^{2}+\right)^{12}}(1-\mathrm{a}-\mathrm{i} \alpha)
$$

Using $\lambda_{n}^{l}$ the other eigenvalues $\mathrm{A}^{\prime \prime}$ are computed in a straightforward manner as

$$
\begin{gathered}
\lambda_{n 1 r}^{n} \sim\left[\frac{\left.(2 n-1)^{2} \pi^{2}, \sqrt{(2 n+1)^{4} \pi^{4}: R^{2}}\right]^{1 / 2}}{2}\right] \\
\lambda_{n \mid i}^{n} \sim \frac{R e}{\sqrt{2}}\left[(2 n-1)^{2} \pi^{2}+\sqrt{(2 n-1)^{4} \pi^{4}+R e^{2}}\right]^{-1 / 2},
\end{gathered}
$$

where $\lambda_{n 1 r}^{u}$ and $\lambda_{n 1 i}^{u}$ are the real and imaginary parts of $\lambda_{n 1}^{u}$. Figure 2 compares the loci of the first three eigenvalues as $R e$ increases as computed from the asymptotic formulae (11) and (13) with their direct numerical calculation from (8); the agreement can be seen to be excellent for $R e>100$. Notice that as $R e \rightarrow$ oo, the $\lambda^{l}$ approach the real axis whereas the $\lambda^{\prime \prime}$ approach the line $\lambda_{r}=\lambda_{i}$. Observe also that the spacing along the real axis between $\lambda_{n}^{l}$ and $\lambda_{n+1}^{l}$ approaches $2 \pi$ whereas the spacing between A" and $\lambda_{n+1}^{\prime \prime}$ approaches $4 n \pi^{2} / \sqrt{\operatorname{Re}}$.This means that given $N$ and $\epsilon>0$, we can find an Re such that $\lambda_{N}^{\prime \prime}$ and $\lambda_{N+1}^{*}$ are within $e$ of each other. This fact has important implications for an eigenfunction expansion at large $R e$; there will be a tendency for the first-quadrant eigenfunclions to behave as if they were almost linearly dependent and consequently for the relevant matrices to become ill-conditioned. Although these facts are not of much relevance to the field driven by an oscillating lid, as $R e$ can practically be at most moderately large, they will be of great relevance to the wave motion situation mentioned in $\S 1$.

(e) As $R e \rightarrow 0$, it can be shown that (8) can be written approximately as

$$
\sin \lambda=-\lambda+\frac{i R e}{2 \lambda} \cos ^{2} \frac{1}{2} \lambda .
$$

Assuming appropriate asymptotic forms for $\lambda_{n r}^{u}$ and $\lambda_{n i}^{u}$ as $\lambda_{n r}^{u}=(4 n-1) \pi / 2+A_{: \mid \text {r }}^{\prime}$ and $\lambda_{n i}^{u}=\ln (4 n-1) \pi+\lambda_{n l i}^{u}$, inserting into (14) and dropping quadratic terms like 

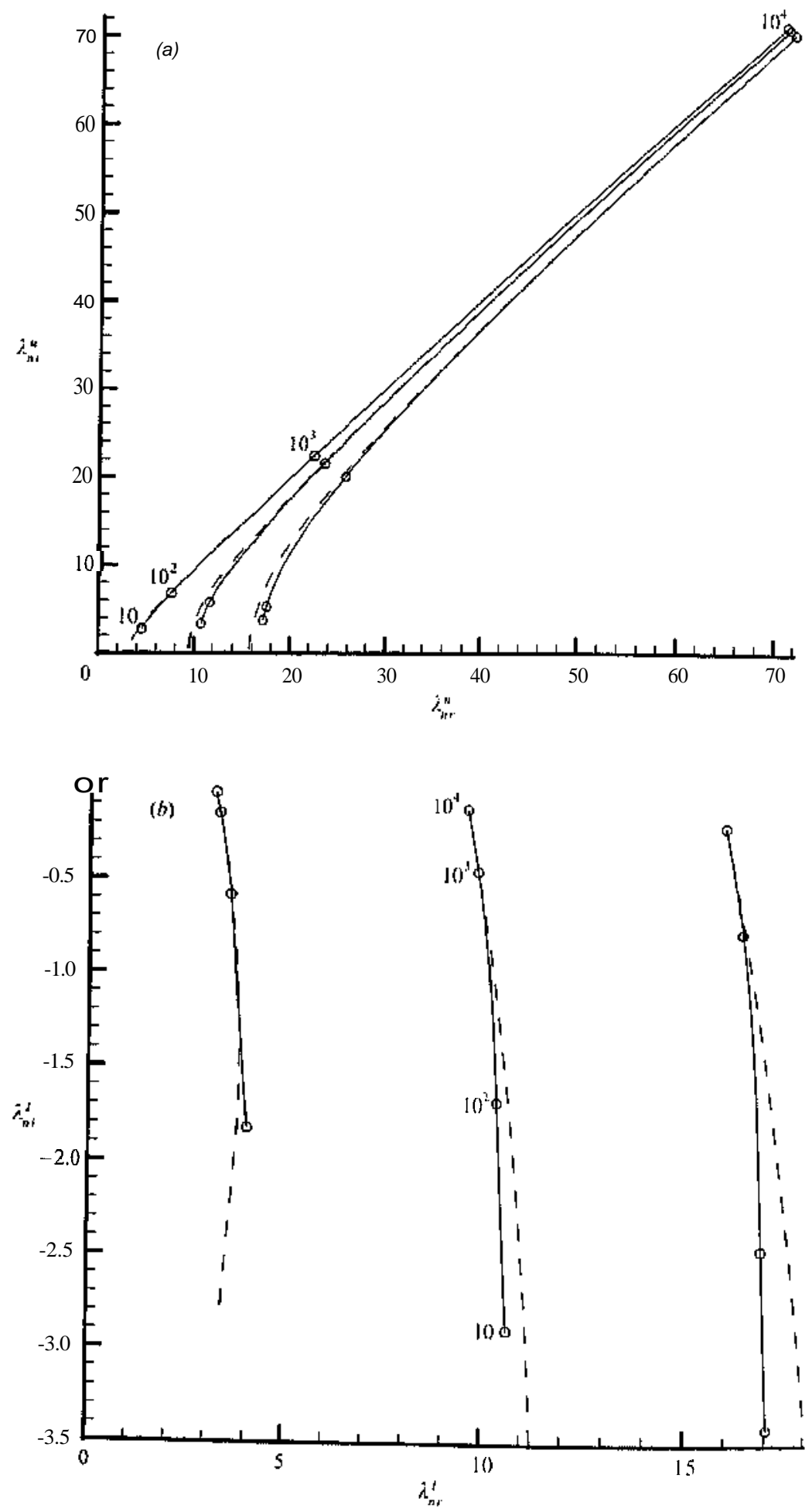

FIGURE 1 The loci of the first three eigenvalues with increasing, Reynnlds number. The circles

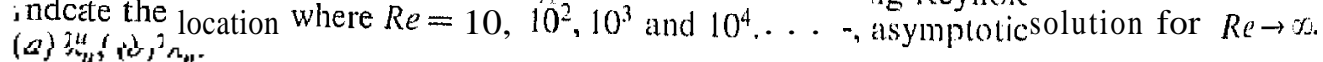


$\hat{i}_{n 1 r}^{u}{ }^{2}$ yields the second-order linear system

$$
\begin{aligned}
& a_{11} \lambda_{n i r}^{u}+a_{12} \lambda_{m 1 i}^{u}=b_{1}, \\
& a_{21} \lambda_{n 1 r}^{u}+a_{22} \lambda_{m 1 i}^{u}=b_{2} .
\end{aligned}
$$

where

$$
\begin{gathered}
a_{11}=\frac{(4 n-1) \pi}{2}(1-\ln (4 n-1) \pi) . \\
a_{12}=-\left[\frac{(4 n-1)^{2} \pi^{2}}{4}+2 \ln (4 n-1) \pi-\frac{R e}{8}(4 n-1) \pi\right], \quad a_{21}=-a_{12}, \quad a_{22}=a_{11}, \\
b_{1}=[\ln (4 n-1) \pi]^{2}-\quad \begin{array}{c}
R_{0} \\
\delta_{0}(4 n-1) \pi, \quad b_{2}={ }_{4}-\frac{(4 n-1) \pi}{2} \ln (4 n-1) \pi .
\end{array}
\end{gathered}
$$

The corrections obtained give eigenvalues which are very good approximations for fixed $R e$, not necessarily small, and large $n$. For example, for $R e=10$. the first eigenvalue $\lambda_{i 1}^{\|}$is approximated to within $3 \%$ whereas the approximation to $\lambda_{10}^{\prime \prime}$ is accurate to within $0.03 \%$. The $\lambda^{l}$ are then obtained from the $\lambda^{\prime \prime}$.

\subsection{The eigenfinction expansion for the velocity field}

We are now in a position to write down a formal expansion for the velocity field in terms of the eigenfunctions described in the previous sub-sections. Recall that we had assumed a form $\psi(x, z, t)=\psi(x, z) \mathrm{e}^{\mathrm{i} t}$ for the stream function. Prior to expanding $\psi(x, z)$ in terms of the new eigenfunctions, it will prove convenient to treat the field as being the sum of a field symmetric in $z$ and of one antisymmetric in it, i.e. $\psi(x, z)=\psi_{s}(x, z)+\psi_{u}(x, z)$ where

$$
\left.\psi_{s}(x, z) \sim f(x) \cosh \right) . z, \quad \psi_{a}(x z) \sim f(x) \sinh \sim / z .
$$

The boundary conditions can also be written as sums of z-symmetric and $z$ antisymmetric parts and consequently the symmetric and antisymmetric fields can be calculated independently of one another. We now write down the formal eigenfunction expansions

$$
\begin{aligned}
& \psi_{s}(x, z)=\sum_{n} a_{n} f_{n}\left(x: \lambda_{n}^{\mu}\right) \frac{\cosh \lambda_{n}^{u} z}{\cosh \lambda_{n}^{u} \frac{1}{2} h}+\sum_{n} b_{n} f_{n}\left(x ; \hat{\lambda}_{n}^{\prime}\right) \frac{\cosh \lambda_{i r}^{l} z}{\cosh \lambda_{n}^{l} \frac{1}{2} h} \\
& \psi_{a}(x, z)=\sum_{n} c_{n} f_{n}\left(x ; \lambda_{n}^{u}\right) \frac{\sinh \lambda_{n}^{n} z}{\sinh \lambda_{n}^{n} \frac{1}{2} h}+\sum_{n} d_{n} f_{n}\left(x ; x_{n}^{l}\right) \frac{\sinh \lambda_{n}^{I} z}{\sinh \lambda_{n}^{l} \frac{1}{2} h} .
\end{aligned}
$$

where the $a_{n}, b_{n}, c_{n}$ and $d_{n}$ are complex scalars that have to be determined from the boundary conditions. The boundary conditions on $z-h / 2$ are

$$
\psi_{v}\left(x, \frac{1}{2} h\right)=\psi_{a}\left(x, \frac{1}{2} h\right)=0, \quad \psi_{s, z}\left(x, \frac{1}{2} h\right)-\psi_{a, z}\left(x, \frac{1}{2} h\right)=\frac{1}{2} u_{0}(x)
$$

Now the scalars can be determined by the least-squares procedure that was described in detail in Shankar (1993). Briefly, we truncate all sums in (16) to $N$ terms, choose $M$ equidistant points on $[0,1 / 2]$ and determine the $4 N$ coefficients in such a way that the boundary conditions are satisfied in a least-squares sense on these points. This has been shown to work very well in many cases in the past and it seems to work even better here. 


\section{Discussion of the results}

There are two parameters, the dimensionless depth of the container $h$ and the Reynolds number $R e$, which enter the unsteady problem as opposed to $h$ alone in the steady case. Calculations show that although $R e$ greatly affects the time scales on which phenomena take place, the qualitative features are not greatly affected, at least for the small $R e$ that we are interested in here. In order to save space we will therefore restrict our attention to the single Reynolds number, $R e=0.1$, with the understanding that the phenomena are not qualitatively very different at other low Reynolds numbers.

Unless stated otherwise, for all the examples considered here $N=20$ and $M=2 N$ : this is a very small value for $N$ and still the boundary conditions are satisfied very accurately as can be seen from the streamlines very close to the boundaries. We have checked these results with $N=100$ and find little difference between the more accurate and less accurate results.

We observe that the dimensionless period $T$, with the normalization that we have chosen, is $2 \pi$ throughout, i.e. $T=2 \pi$, and we need only observe the field over a single period $0 \leqslant t \leqslant T$. In fact, we need only limit our observations to a half period $0 \leqslant t<T / 2$ since over the other half only the flow direction changes with identical corresponding eddy structures. We will therefore limit our observations to $0 \leqslant t<T / 2$.

\subsection{Periodic eddies in an infinitely deep container}

We shall first consider a somewhat simplified case, where the container is very deep and the bottom has no impact on the field. In this case alone, the origin is assumed to be at the intersection of the plane of symmetry with the lid and $z$ still points upwards. Now we need only retain those eigenvalues which have positive real part and the expansion (16) now simplifies to

$$
\psi(x, z)=\sum_{n} a_{n}^{\infty} f_{n}\left(x ; \lambda_{n}^{n}\right) \exp \left(\lambda_{n}^{\prime} z\right)+\sum_{n} b_{n}^{o} f_{n}\left(x ; \lambda_{n}^{l}\right) \exp \left(\lambda_{n}^{\prime} z\right)
$$

where only two sets of coefficients $\left\{a_{n}^{\infty}\right\}$ and $\left\{b_{n}^{\infty}\right\}$ need to be determined from the boundary conditions on the lid: for a given $N$ the number of complex unknowns is $2 N$.

The oscillatory instantaneous streamline patterns in such a container are shown in figure 3 at four different times over the half period. The fields are shown only to a depth of three container widths; and only in the range $0 \leqslant x<0.5$ since there is symmetry about the plane $x=0$. There is little change in the patterns up to $\mathrm{f}=T / 8$ but when $t=T / 4$ one can clearly see that the first primary eddy has shortened considerably and the streamlines around the primary eddy centres have a more circular appearance. At $t=3 T / 8$ the patterns look like those at the earlier times but the direction of the circulation has now reversed. Note that the plate is stationary at $t=774$ and reverses direction for $t>T / 4$. Thus $u(x, 0, t)$ would have to vanish at the former time and reverse direction at the latter and this is indeed what happens. A question of considerable interest, perhaps the most interesting in this investigation, is: how exactly does the change from $(c)$ to $(d)$ lake place? The first primary eddy cannot simply change direction instantaneously; in fact, some other eddy mechanism must operate to moderate this drastic change. We will discuss this in greater detail in $\S 3.3$.

We can say something definite about the nature of the eddies seen in figure 3 as I $z \mid \rightarrow \infty$. In this limit the eddy structure will be dominated by the nature of the 
(a)

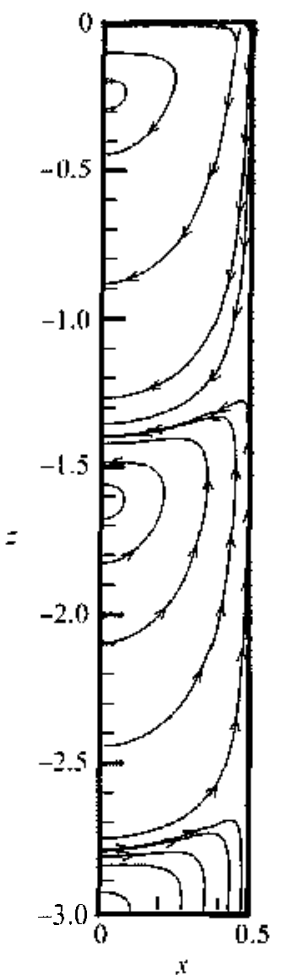

(b)

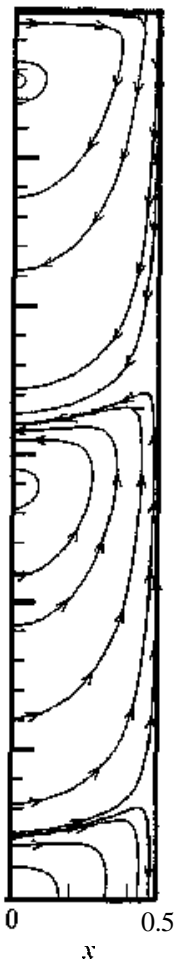

(c)

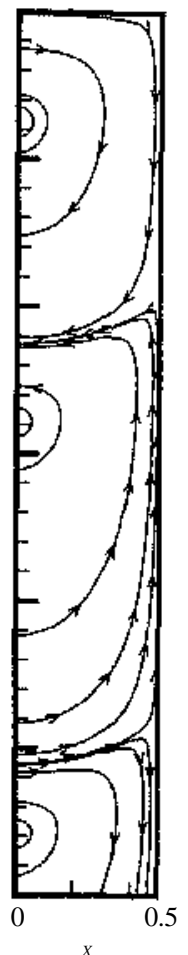

(d)

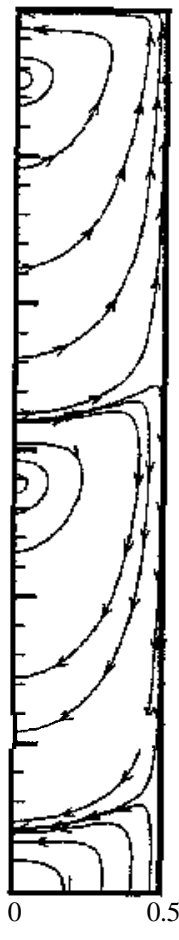

FiguRE 3. Oscillatory streamline patterns in an infinitely deep container $R t^{\prime}=0.1$. (a) $t=0,(b) t=T / 8,(c) t=T / 4,(d) t=3 T / 8$.

eigenvalue with the smallest real part. Looking at table 1 it is clear that the dominant eigenvalue is $\lambda_{1}^{l} \approx 4.2099-2.2461 \mathrm{i}$, although the real part of $\lambda_{1}^{u}$ is only slightly larger. Thus for large $z \backslash, \psi$ is determined by the first terms alone in the series (18). Since the $z$-dependence is given by, say, $\exp \left(\lambda_{1 r}^{l}+i \lambda_{1 i}^{l}\right) z$, the counter-rotating eddies will be spaced $\sim \pi / \lambda_{1 i}^{l} \approx 1.399$ apart while the field will decay by a factor of $\exp \left(-\pi\left|\lambda_{1,}^{l} / \lambda_{1 i}^{l}\right|\right) \approx 1 / 361$ going from one eddy centre to the next. It may be observed that the eddy spacing is already close to the asymptotic value in figure 3.

\subsection{The general eddy structure in containers of finite depth}

When dealing with cavities of finite depth we have to use the full expansions as given in (16). Most aspects of the general eddy structure and its dependence on the depth $h$ can be inferred from the instantaneous streamline patterns seen in figures 4-7. In each figure the patterns are shown at times spaced $T / 8$ apart. In the shallowest container of depth $h=0.2$, figure 4 , the general ciruculation is, as might be expected, clockwise until $t=T / 4$; however the primary eddy has two secondary eddies inside it, one on each side of the symmetry plane, connected by a saddle which is not shown. This secondary structure changes in size over the period as can be seen from the figure. Figure $4(c)$ also shows the existence of a corner eddy at the quarter-period which cannot be seen at the other times. Once again we note that the circulation changes sign beyond this time, as it must since the plate motion has now changed direction. 

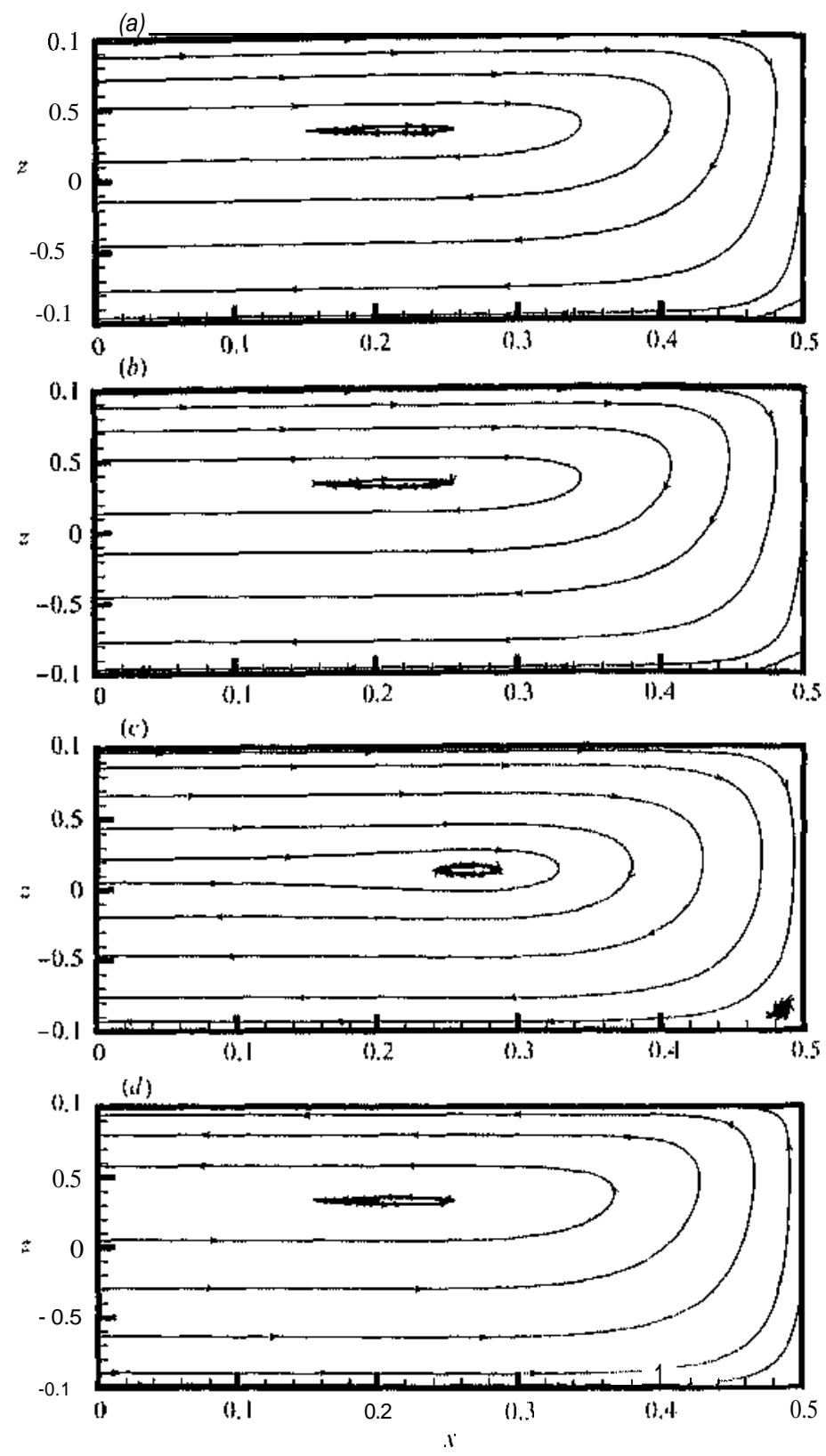

Figure: 4. Periodic streamline palleriss in a comtainer of depth $/ \mathrm{h}=0.2, R e=0.1$. (a) $t=(),(b) /=7 / 8,(c) 1=7 / 4$. (l) $/ \ldots 3 \% / 8$.

Note that we do not show any pallerns at the hall-period since this would be identical to those at the initial instant but with the circulation reversed.

The situation is somewhat different in a container of unit depth, ligure 5. Here the single primary eddy has no secondary structure; in liact,al each in stant shown the flow field looks qualitatively like the flow liekl that one would find in stcadyflow (Shankar 1993). A comer eddy can be seen in each of the frames but its size and orientation changes with time. At the quarter-period, when the plate is instantaneously at rest, 
(a)

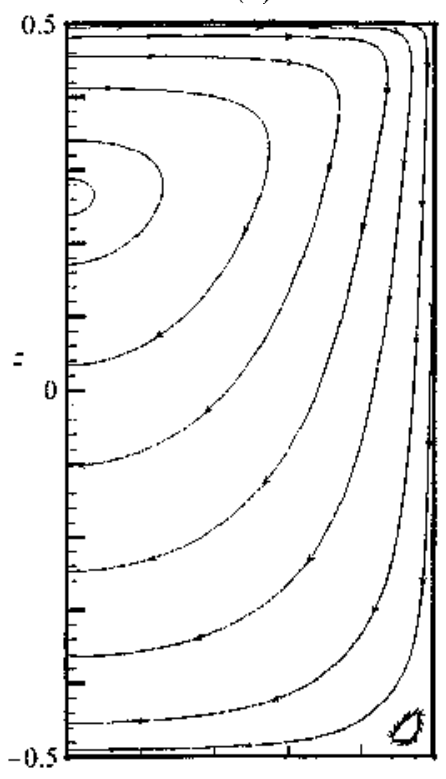

(c)

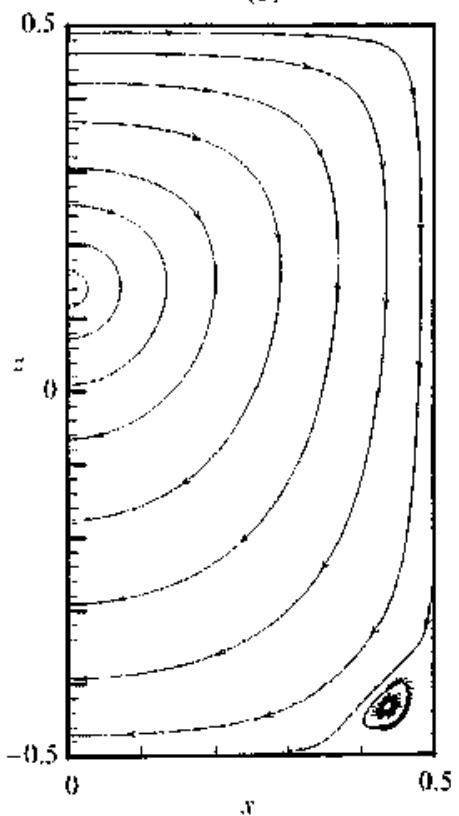

(b)

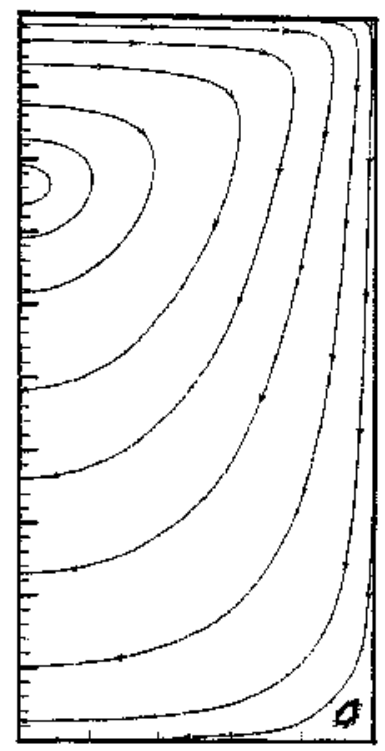

(d)

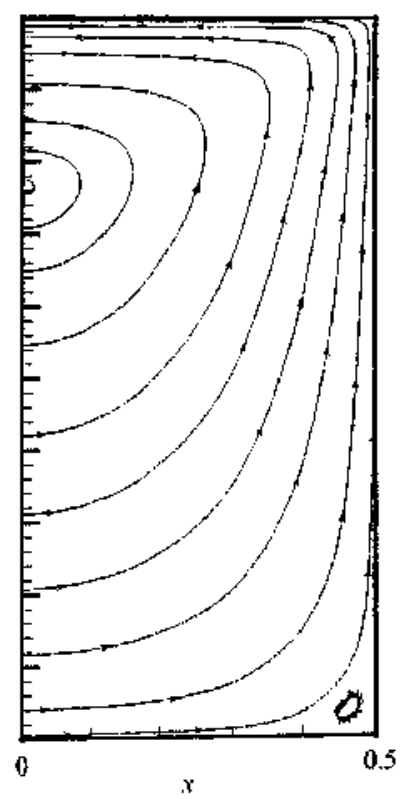

FIGURE 5 Periodic streamline patterns in a container of depth $h=1.0 \cdot R e-0.1$. (a) $t=0,(b) t=T / 8$, (c) $t=T / 4$, (d) $t=31 / 8$.

the central streamlines tend to be more circular and overall the pattern looks different from that seen earlier. An eighth of a period later the direction of the circulation has reversed, figure $5(d)$. We will examine this transition more carefully in the next sub-section.

Examining figure 3 we conclude that when the container depth is around the asymptotic eddy spacing a second primary eddy should be formed over the cycle. 
(a)

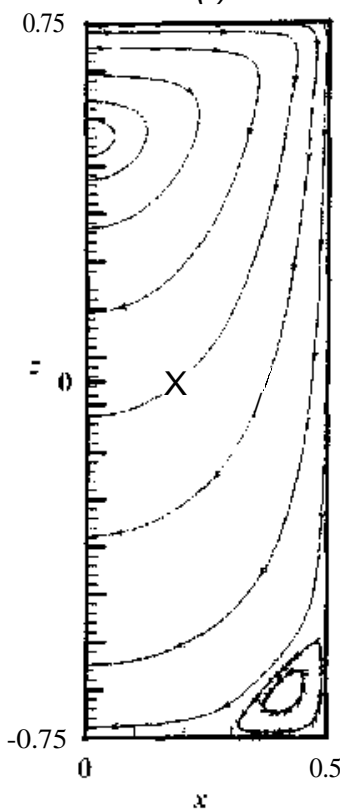

$(b)$

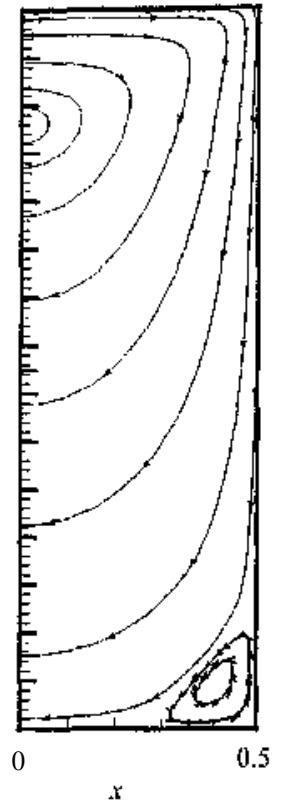

$(c)$

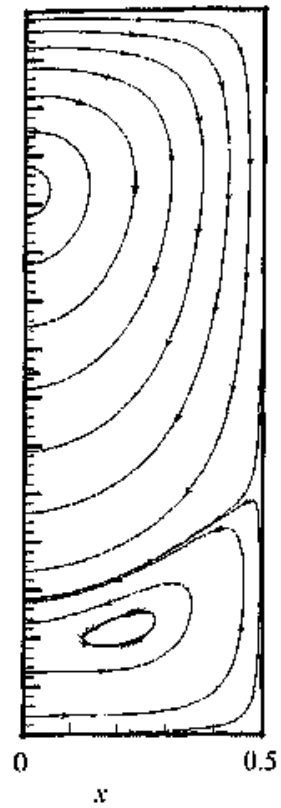

(d)

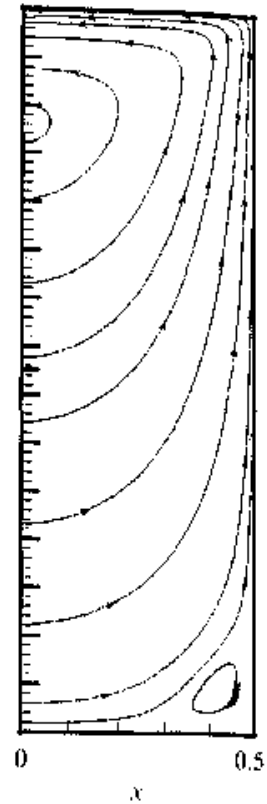

FIGURE 6. Periodic streamline patterns in a container of depth $/ ?=1.5, R e^{2}=0.1$. (a) $!=0$, (b) $t=T / 8,(c) t=T / 4,(d) /=3778$.

This is seen in figure 6 for a container of depth $h=1.5$. In the first two frames we observe just a single primary eddy and a large corner eddy. But at the quarter-period the primary eddy is compressed in size with its streamlines more circular. Below it is a second counter-rotating primary eddy. This eddy contains within it a secondary structure of two eddies whose centres are connected by a saddle on the symmetry plane. We are not able to resolve a corner eddy at this instant. At $t=3 T / 8$ the circulation has reversed, the secondary eddy has disappeared and the corner eddy has reappeared but with its circulation reversed. We will examine the formation of the second primary eddy in $\$ 3.4$.

Finally, as an example of a deep container we consider the case of $h=4$ in figure 7 . There are generally three primary eddies in this case, as might have been guessed from the value of approximately 1.4 for the asymptotic spacing of the eddies. We observe that there are corner eddies at all the times shown. As in $\$ 3.1$ the main change seen is the compression of the first primary eddy and the clear distortion of the central streamlines to a circular shape at the quarter-period. Once the circulation reverses, the streamline pattern is much like the earlier pattern.

\subsection{The mechanism for the reversal of the general circulation}

Perhaps the question of greatest interest in this investigation concerns the nature of the reversal of the general circulation over each half-cycle of the lid motion. It is noted in each of figures 4-7 that at $t=T / 4$, when the lid is instantaneously at rest, the circulation in the first primary eddy is always clockwise. At $t-3 T / 8$ the circulation is anticlockwise following the lid motion. This change could not have been instantaneous and we wish here to examine how exactly this change takes place. 
(a)

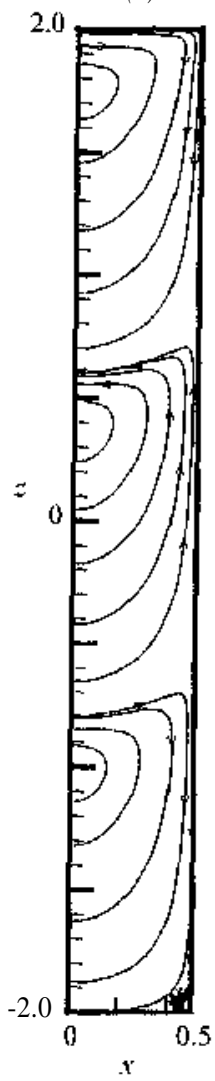

(b)

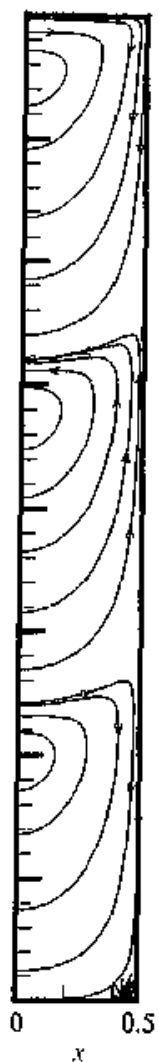

(c)

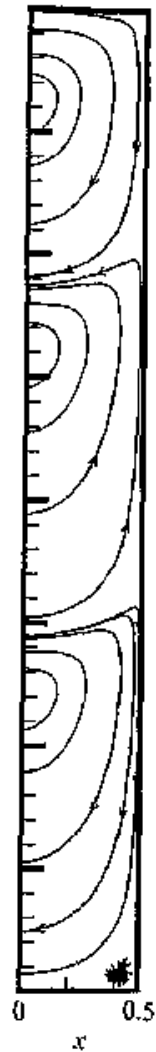

(d)

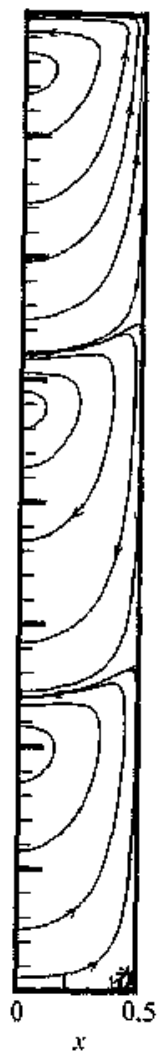

FIGURE 7. Periodic streamline patterns in a container of depth $h=4.0, R e=0.1$. (a) $t=0$, (b) $t=778,(c) t=T / 4,(d) t=3 T / 8$.

Figure 8 presents details of the transition of the flow pattern when the lid reverses its direction of motion. Before the reversal, the flow pattern is similar to the one in figure $5(\mathrm{a})$ and consists of a large primary eddy $\mathrm{A}$ and a corner eddy $\mathrm{C}$ with a zero streamline ZS1 dividing the two. Now when the lid starts to move in the opposite direction, a thin region of fluid near the lid starts to circulate in a sense opposite to the bulk due to the non-slipping of fluid on the lid (figure $8 a$ ). This eddy, $\mathrm{B}$, contains two centres, one on the left and another on the right of the symmetry plane and the two centres are connected by a saddle on this plane. This whole structure mediates between the large primary eddy whose circulation is now in the "wrong" direction and the lid which is now moving to the left. It is clear that there must be a zero streamline ZS2 dividing the two circulating regions. With increasing time the substructure in the new primary eddy B shrinks until the two centres and saddle coalesce to a single centre on $x=0$. Within a ten-thousandth of a time-period, regions $\mathrm{B}$ and $\mathrm{C}$ increase rapidly in size while region A shrinks (figure 8b). This continues with ZS1 and ZS2 drawing ever closer to each other (figures $8 c, d$ ) till they finally touch, two saddles S1 and S2 appear and a saddle-saddle connection or heteroclinic orbit is formed (figures $8 e, f$ ) (S2 is not seen as only one half of the container is presented throughout). At this stage, there is a zero streamline ZS3, not shown in figure $8(e)$, separating region A (which is by this time the same size as B) and C. Though this is not visible in the 


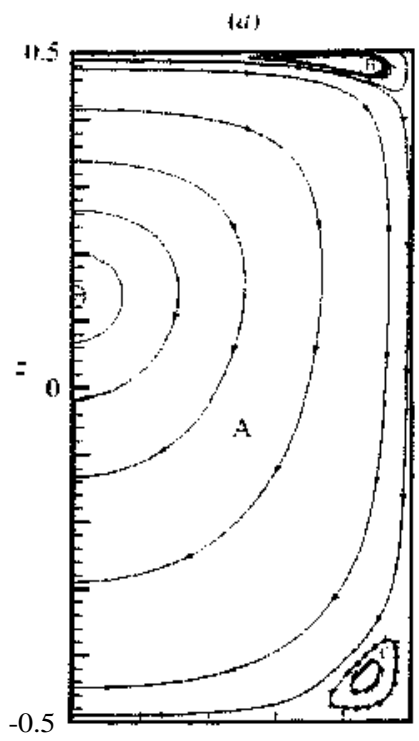

(h)

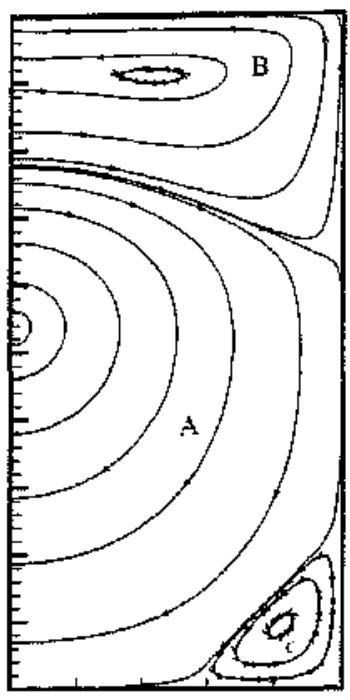

(i')

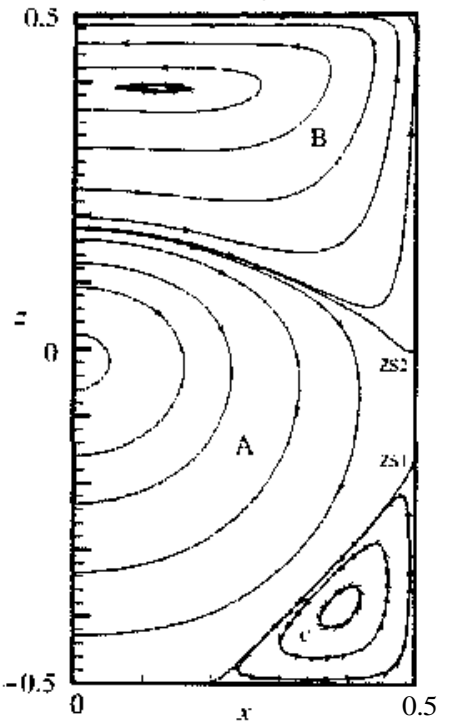

(di)
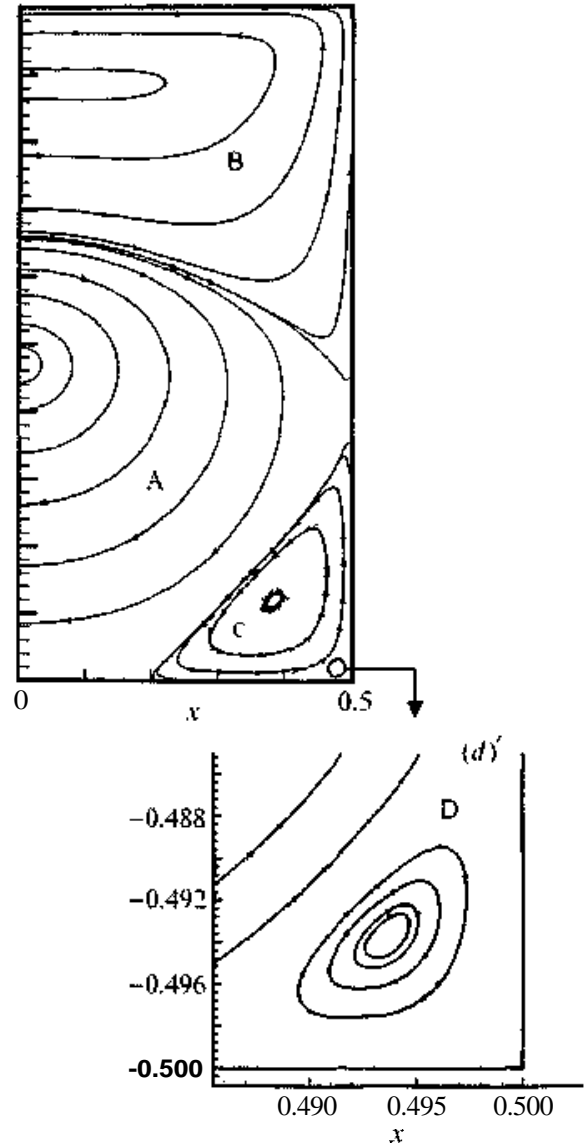

FIGURE $8(a-d)$. For caption see facing page. 
(c)

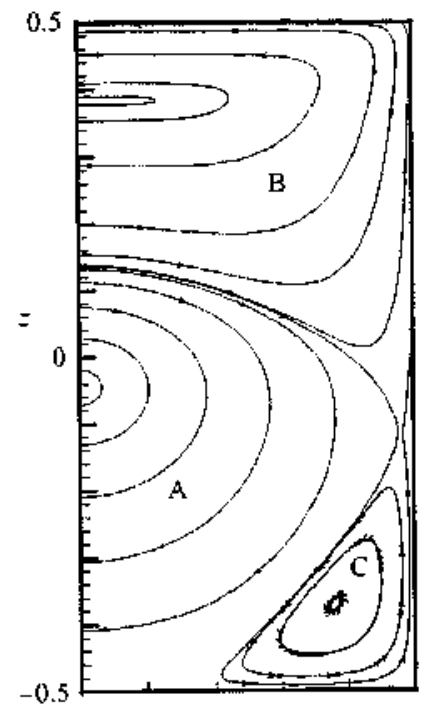

(g)

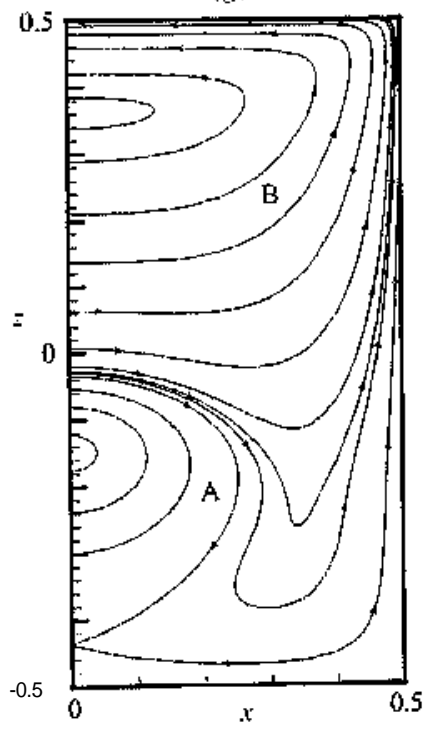

(f)

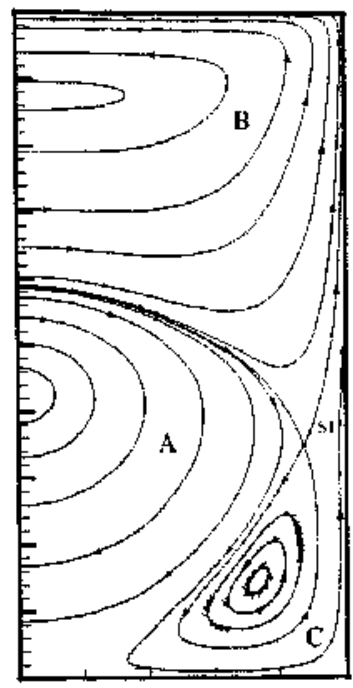

(h)

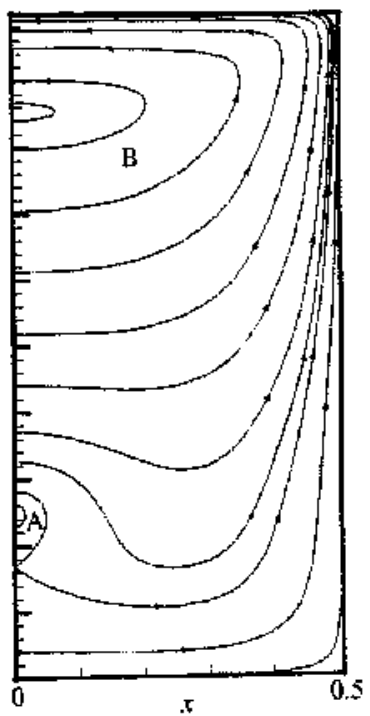

Figube ${ }^{8}$. Details of the transient eddy structure during flow reversal for $t>T / 4 ; h=1.0$. $R e=01$ (a) $\mathrm{r}=0.25001 T,(b) t=0.25015 T$, (c) $t=0.25020 T$, (d) $t=0.250217$, (e) $t=0.25022 T$ $(/) ?=0.250247,(\mathrm{~g}) t=0.25028,(h) \mathrm{r}=0.25031 T$.

figure there is also a new corner eddy D with a sense of rotation opposite ${ }_{\text {to }} \mathrm{C}$ with another zero streamline ZS4 dividing the two. The two end's of ZS3 meanwhile $r_{-_{r_{--}}} y$ approach each other and touch forming a limacon, the saddles S1 and S2 disappear together with the heteroclinic orbit and what was once the comer eddy $\mathrm{C}^{\prime \prime}$ has now been engulfed by the new primary eddy B (figure $8 g$ ). Meanwhile region A con inues to shrink and is on the verge of disappearing in the firnal panel, figure $8(h)$. The flow topology subsequent to this time will be similar to that in figure $5(d)$. The transition 
(a)

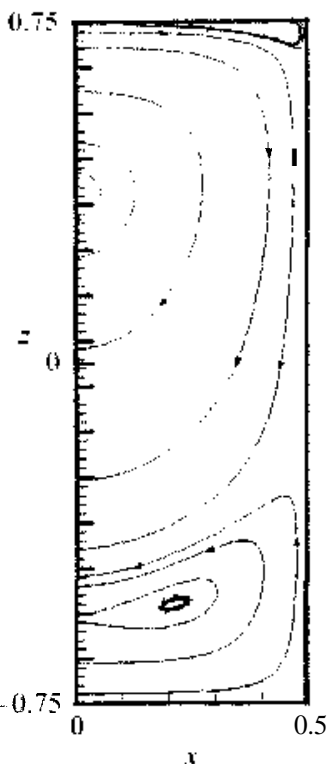

(th)

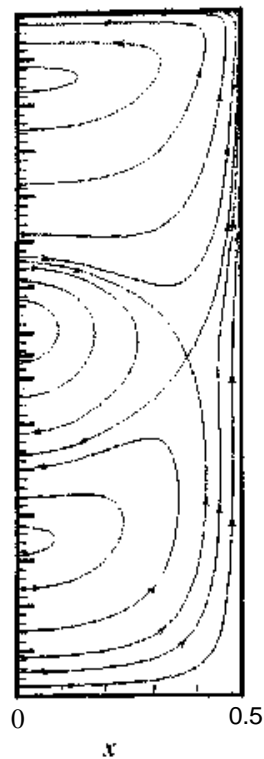

(c)

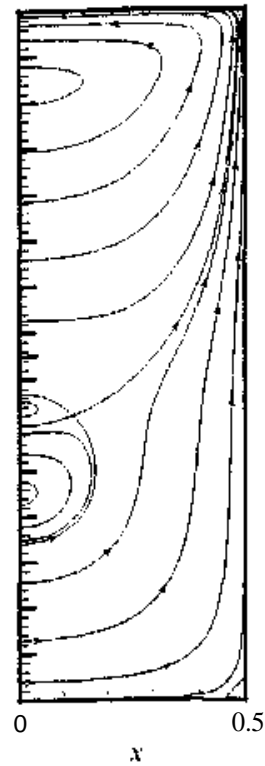

(i)

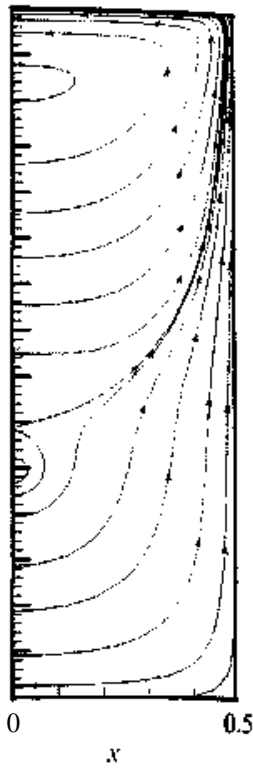

FIGURE 9. Details of the transient eddy structure during flow reversal for $t>0.25 T: h=1.5$, $\operatorname{Re}:=0.1$. (a) $t=0.25001 T$, (b) $t=0.250307$, (c) $t=0.25037 T$, (d) $t=0.25038 \mathrm{~T}$.

time from the initial to the final flow pattern is roughly three ten-thousandths of a time period.

Figure 9 shows similar details for the case of a deeper container when $h=1.5$. The transition details are similar to $h=1.0$ except that the specific flow topologies that occur in the transition are more complicated due to the presence of three primary eddies compared to two for $\mathrm{ft}=1.0$ (figure 9). For example, there are four heteroclinic orbits in figure $9(b)$ compared to two in figure $8(f)$. It is clear from the transitions shown here that, twice over each cycle as the direction of motion is reversed, there is a tendency for good mixing to take place. However, since these changes occur over a small fraction of the period, at least in the cases shown here, one may wonder whether this contribution to the mixing process can be significant. We will discuss this matter in greater detail later when some other factors will also be considered.

The results shown here are all for $R e=0.1$. We find very similar qualitative results for higher Reynolds numbers but as $R e$ increases the time scales, as fractions of the period. over which the transition takes place continue to increase. In $\$ 3.6$ we will discuss the results obtained for $R e=100$ where the transition time is a significant fraction of the period.

\subsection{The formation and decay of the second primary eddy, $\mathrm{ft}=1.5$}

Another transient phenomenon of interest is the appearance and disappearance of the second primary eddy seen in figure 6 . We see in figure $6(\mathrm{c})$ a second counterrotating primary eddy but only a single primary in the other panels. The details of the formation of the second primary eddy from merger of the corner eddies can be seen in figure 10 . 
$\{a\}$

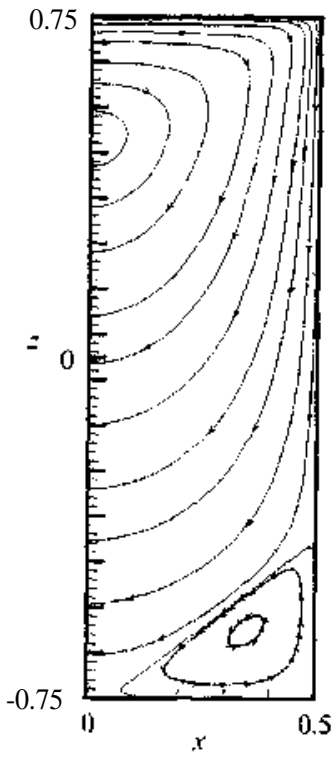

(h)

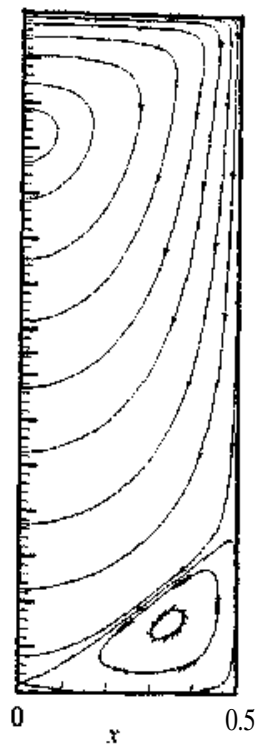

$(c)$

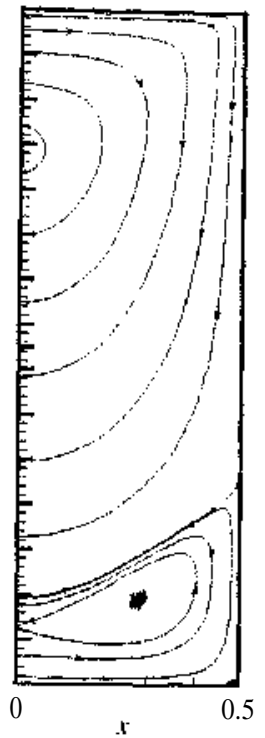

FIGURE 10. Formation of the transient second primary eddy from the merger of the growing corner eddies $h=1.5, R_{\underline{e}} \quad 0.1 .(a) \pm 0.2492 T(, b) t=0.2493 T .(c) t=0.2498 T$.

In figure $10(a), t=0.2492 T$, the corner eddy is much larger than it was at $\mathrm{r}=0.125 \mathrm{~T}$ (figure $6 h$ ). With increasing time the two corner eddies keep growing until at around $t=0.2493 T$, figure $10(b)$, the corner eddies just touch. With further increases of time the stagnation point on the bottom wall lifts off with a saddle connecting the two centres which together with their surrounding circulating fluid form part of the second primary eddy. This whole picture is very reminescent of what happens in a cavity with a steadily moving lid if the depth is increased (Shankar 1993). Here increasing time is analogous to increasing depth in the steady case. The disappearance of this eddy with further increasing time follows a similar course, though in reverse.

\subsection{Comer eddies}

A matter of theoretical importance is the existence of eddies near sharp corners in viscous flows. For steady flows, Moffatt (1964) long ago showed that, in general, there could be an infinite number of counter-rotating eddies, of decreasing size and strength, near a corner. Moffatt was able to employ a simple similarity analysis of the biharmonic equation in polar coordinates to reach this important conclusion. It appears that no such result is currently available for the linearized, unsteady Navier-Stokes equations (1). In fact, if a Moffatt-type analysis is attempted on (2) we soon find that because the operator in (2) is not fully homogeneous in $r$, the radial coordinate, no simple resolution of the question is possible. In this situation the evidence available from the flow fields computed here takes on some importance. We note the primary corner eddies can be seen in all figures 4-7. although they are absent in some of the panels of figure 4; even in the latter case these eddies could be so small that we have failed to resolve them. So there is a very strong case for the existence of primary corner eddies. We then tried to see whether second corner eddies could be identified. In many of the cases, with $N=300$, second corner eddies could be found but even with 300 eigenfunctions these could not be resolved very well. 
(a)

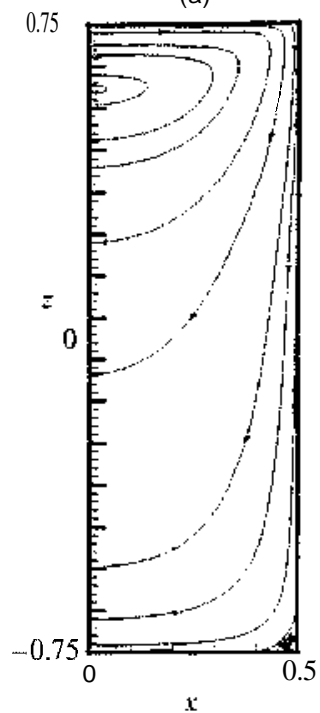

(b)

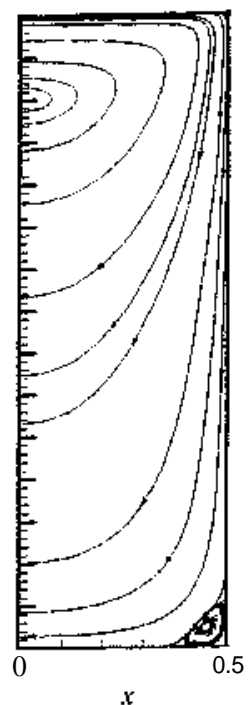

(c)

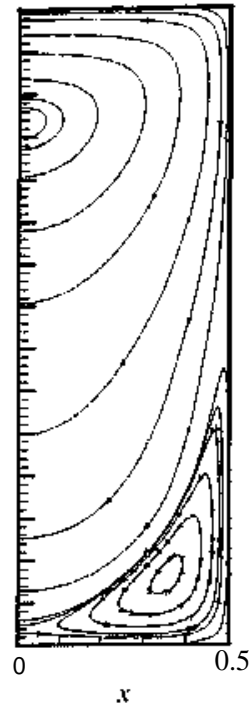

(d)

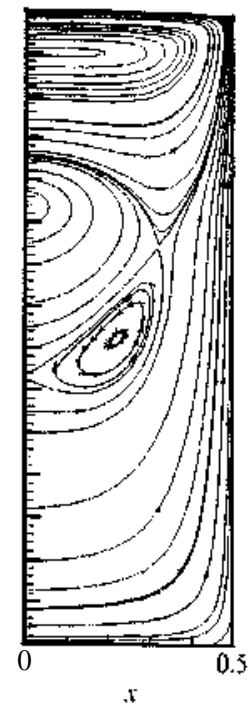

FIGURE 11 Instantaneous streamlines in a container of depth $/ ?=1.5$ at a higher Reynolds number, $R e=100$. (a) $\mathrm{r}=0,(b) t=T / 8,(c) t=T / 4,(d) t=3 T / 8$.

Often it is easier to resolve the weaker eddies when the primary ones grow in size. Thus in figure $8(d)$ we are able to resolve the second counter-rotating corner eddy fairly well because the primary one has grown large in size. But even in this case we are not able to obtain streamlines of this eddy closer to the lower wall because of the errors made in satisfying the no-slip condition. In summary, there is some evidence presented here for the existence of multiple corner eddies although firm conclusions cannot be drawn.

\subsection{Moderate Reynolds numbers}

The oscillatory Reynolds number appears in the analysis here as a parameter that can take all values in the range $0<R e<\mathrm{GO}$. At the low end, the flow is quasi-steady as the viscous term almost balances the pressure and the fluid responds almost instantaneously to the lid. This is the Stokes regime. At the other end, linearizing the Navier-Stokes equation by dropping the convective terms needs justification. In flow situations involving just a translational velocity scale, it would indeed be wrong to drop these terms at high $R e$. However, in a situation like the present where two Reynolds numbers can be defined, the linearization is justified provided $\mathrm{Sr} \gg 1$. We can, therefore, consider applying our procedure to flows where the Reynolds number is moderately large with the clear understanding that $R e_{t r}$ is kept sufficiently small.

Figure 11 shows the eddy structure for a moderately large $R e=100$ and $h=1.5$. The eddies are flatter near the lid and the eddy centres are located closer to the top wall for $R e=100$ than for $R e=0.1$ (figure 6 ). Consequently the velocity gradients in the $z$-direction near the lid are steeper. Thus one already sees the formation of unsteady boundary layers near the walls. Compared to what is seen in figure 6, the corner eddies are, prior to flow reversal, much smaller, presumably because of the larger momentum flux down the wall. However, at $/=T / 4$, when maximum deceleration occurs, the corner eddy penetrates to a greater extent into the primary eddy than for the $R e=0.1$ case (figure $6 c$ ). The latter case has reached a quasi-steady 
state by $t=3 T / 8$ (figure $6 d$ ), whereas for $R e=100$, the flow is still in transition with complicated structures (figure $11 d$ ) appearing and disappearing. Similar features and time scales hold for the container of unit depth. If we recall that transition occurred in about $0.0003 T$ at $R e=0.1$ while now at $R e=100$ it occurs in something over $0.125 T$. we may conclude that by increasing the Reynolds number we can significantly increase the period over which this transition may take place.

We will now assess a number of factors that have arisen here that have implications for the study of mixing. Following Aref (1986), we will mean by 'mixing' the process of diffusion of substance across intermaterial surfaces, in contrast to stirring which is a process of stretching of intermaterial area. Of course, stirring contributes "to mixing by increasing the intermaterial surface area. Mixing is often synonymously used for stirring in the literature (e.g. Ottino 1989) but here we will maintain the distinction. For two-dimensional incompressible flows, it is well-known that stirring is poor in steady flows and better when the boundaries operate in a periodic manner (Ottino 1989). This is because the equations of motion of an advected particle in such flows form a 1-degree-of-freedom Hamiltonian system with the stream function $\psi$ being the Hamiltonian. Such systems are integrable if $\Psi$ is independent of time (in which case the streamlines are the pathlines) and can exhibit chaotic behaviour if $\psi$ is a function of time. Chaotic advection is known to cause efficient stirring (Aref 1984). In dynamical systems theory, one way to study chaos has been to perturb an integrable system. Hamiltonian systems with homoclinic and heteroclinic loops are known to be good candidates for generating chaos when perturbed; breaking of these loops is a well-established mechanism to produce chaos in Hamiltonian systems (e.g. Wiggins 1996). In the present context, the steady Stokes flow provides the unperturbed system (where the particle motion is integrable) with the perturbation being a modulation in time of the stream function (usually by varying the boundary conditions). If the modulation is sufficiently slow, the inertia terms can be dropped (Aref \& Balachandar 1986) and $\Psi$ satisfies the biharmonic equation. The time dependence of $\Psi$ enters only through the boundary conditions and this is enough to produce chaotic advection and attendant stirring. The cavity mixing experiments presented in Ottino (1989) also fall in the Stokes regime in: $R e_{t r}, R e_{u s} \approx O(1)$ in these experiments. Again, good stirring is observed.

In the present study, we have explored the implications of retaining only the unsteady inertia term. As we have shown, this is meaningful if the Strouhal number. $S r \gg 1$. Even though the velocity field is unsteady and the particle motion probably chaotic (this would have to be established by standard chaos diagnostics like Poincare maps), the stirring will be good only if the oscillatory Reynolds number $R e$ is small; however the extra mixing due to truly unsteady effects around $t=T / 4$ and $t=3774$ will be small because the fraction of the period over which it is effective is small. If we now increase the frequency, thus increasing $R e$, this fraction increases and so this tends to promote increased mixing. We conjecture that the local mixing in regions where the flow is changing rapidly may be good. A case in point is the sweeping up of fluid in the normally stagnant lower corners into the main body of the fluid, twice over each cycle. This occurs even for a low $R e=0.1$ (figure $8 e-g$ ) even though the fraction of time over which this happens is small. A traditional quasi-steady analysis would have missed this completely. A blob placed in the corner would have been thought to remain there; the present analysis shows this is not really true. If the Reynolds number is moderate, the fraction of the period over which the truly unsteady fields contribute to the mixing increases considerably. However, since $£ 2$ has to be increased for $R e$ to increase, the stirring will tend to be reduced. This is because the more rapid 
oscillations of the container leave less time for the particles to advect appreciably. A material line just does not have enough time to be stretched and folded globally even though the particle motion may be chaotic. It could well be that there is a range over which both effects could be optimized; this can only be determined by a detailed analysis of the mixing process. Stirring would improve if we could weaken the restriction $S r \gg 1$ by increasing the lid translational velocity $U$; in practice we could do this with the clear understanding that the present analysis will only provide approximate estimates of the actual field in this case (as the nonlinear convective terms have been ignored). This is not unreasonable since for steady laminar flows in cavities the Stokes flow approximation provides a good approximation even for moderate Reynolds numbers; only features such as the lack of fore-aft symmetry are missed. Thus although only a comprehensive analysis of mixing, taking into account all major factors, can provide a fully reliable picture the present calculations suggest that unsteady effects can indeed enhance the local mixing.

The situation is quite different if we wish to investigate viscous effects on wave motion in a container. Consider small-amplitude wave motion in the container which now has no lid and where the liquid free surface is at $z=0$. If the Reynolds number is high, to a first approximation the surface-gravity wave natural frequencies can be computed from an inviscid analysis. The viscous corrections to the frequencies and the estimation of viscous damping depend on a solution of the linearized Navier-Stokes equations (1) (Nicolas 2002). Here, the boundary conditions at $-=0$ are the interface kinematic condition and the continuity of the stress rather than the no-slip condition. In this case the high Reynolds number limit is physically meaningful and is what is of interest. It was with the wave motion connection in mind that the analysis of the spectrum in $\$ 2$ was carried out over the whole range of Reynolds numbers. We expect these results to be of value in the viscous analysis of wave motion.

\section{Conclusion}

We have, in this paper, analysed the periodic motion of a liquid in a rectangular container, generated by the oscillatory motion of the lid. The eigenfunction expansion procedure was used. It was shown that two sets of eigenvalues $\left\{\lambda_{n}^{u}, n=1,2, \ldots\right\}$ and $\left\{\lambda_{n}^{l}, n=1,2, \ldots\right\}$ exist for the eigenvalue problem (3)-(5). They are functions of $R e$ and related through (9). In the limit $R e \rightarrow \infty$, the $\mathrm{A}\{$, approach the real axis and are approximately equally spaced whereas the A" approach the $\lambda_{i}=\lambda_{r}$ line in the complex- $\lambda$-plane and the distance between them decreases.

As might be expected, at low Reynolds numbers the flow field is quasi-steady and resembles the flow in a container driven by a steady lid motion of velocity equal to the instantaneous velocity of the lid. Thus quasi-steady eddy structures A and $\mathrm{B}$ (figure 8) form when the lid is accelerating and decelerating the least. A and B have the same structure but with a reversed flow direction. The transition time $T_{t r}$ for A to change to $\mathrm{B}$ increases with increasing $R e$, from a few ten-thousandths of a period at $R e=0.1$ to almost an eighth of a period at $R e-100$. The flow process occurring during the transition time $T_{r r}$ is truly unsteady and cannot be captured by the steady Stokes equation. During the transition there are features that could considerably enhance local mixing of the fluid. Morever, there is a strong tendency for the fluid in the corner regions to be swept into the main body of fluid. Although only a detailed analysis of the mixing process can determine the parameter regimes where these effects will be significant, there is little doubt that a traditional quasi-steady analysis will underestimate the mixing process. 
Finally it should be pointed out that the present analysis provides a foundation for the analysis of linearized, viscous wave motion in a rectangular, brimful container. The eigenvalues and eigenfunctions derived here can be used directly. The main difference from the analysis of this paper will be that, instead of the no-slip conditions at the lid, the kinematic and stress conditions will have to be applied at the free surface. For this application the asymptotic results for high Reynolds number will be useful.

We would like to thank our colleague Ms D. Shobha for help in preparing the figures.

\section{REFERENCES}

ANDERSON, P. D., GALAKTIONOV, 0. S., PETERS, G. W. M., VAN DE VOSSE, F. N. \& MEIJER. H. E. H. 1999 Analysis of mixing in 3-D time periodic cavity flows. J. Fluid Mech. 386, 149-166. AREF, H. 1984 Stirring bychaotic advection.J. Fluid Mech. 143, 1-21. ARef, H. \& Balachandar, S. 198chaotic advection in a Stokes flow. Physfluids 29, 3515-3521. BATCHELOR, G. K. 1967 An Introduction to Fluid Dynamics. Cambridge University Press.

CASE, K. M. \& PARKINSON, W. C. 1957 Damping of surface waves in an incompressible liquid. J. Fluid Mech. 2, 172-184.

GALAKTIONOV. O. S., Meleshro, V. V., PETERS, G. W. M. \& MEIJER. H. E. H. 1999 Stokes flow in a rectangular cavity with a cylinder. Fluid Dyn. Res. 24, 81-102.

HAPPEL, J. \& BRENNER, H. 1973 Low Reynolds Number Hydrodynamics. Noordhoff.

MALYUGA, V. S., MF.LESHKO, V. V., SPEETJENS, M. F. M., CLERCX, H. J. H. \& VAN HEUST, G. J. F. 2002

Mixing in the Stokes flow in a cylindrical container. Proc. R. Soc. Lond. A 458. 1867-1885. MiLes. J. W. 1967 Surface-wave dampingin closed basins. Proc.R. Soc. Lond.A 297, 459-475. Moffatr, H. K. 1964 Viscous and resistive eddies near a sharp corner. J. Fluid Mech. 18. 1-1 S. NicoLás. J A. 2002 The viscous damping of capillary-gravity waves in a brimful circular cylinder. Phys. Fluids 14, 1910-1919.

Ortino. J. M. 1989 The Kinematics of Mixing: Stretching. Chaos. and Transport. Cambridge University Press.

OTTINO, i. M. 1990 Mixing, chaotic advection and turbulence. Ammu. Rev. Fluid Mech. 22, 207253. ShANkaR, P. N. 1993 The eddy structure in Stokes flow in a cavity. J. Fluid Mech. 250, 371-383.

ShaNKar, P. N. \& DESHPANDE, M. D. 2000 Fluid mechanics in the driven cavity. Annu. Rev. Fluid Mech. 32, 93-136.

STURGES, L. D. \& JOSEPH, D. D. 1977 The free surface on a simple fluid between cylinders undergoing torsional oscillations. Part III : Oscillating planes. Arch. Rat. Mech. Anal. 64, 245-267. WIGGINS, S. 1996 Introduction to Applied Nonlinear Dynamical Systems and Chaos. Springer. 